\title{
Detection and genetic analysis of a novel atypical porcine pestivirus from piglets with congenital tremor in Japan
}

\author{
Miwako Kasahara-Kamiie ${ }^{1}$, Mitsuo Kagawa ${ }^{2}$, Mai Shiokawa ${ }^{3}$, Fujiko Sunaga ${ }^{1}$, Yuka \\ Fukase $^{1}$, Naoyuki Aihara ${ }^{1}$, Takanori Shiga ${ }^{1}$, Junichi Kamiie ${ }^{1}$, Hiroshi Aoki ${ }^{3}$, and Makato \\ Nagai $^{1}$ \\ ${ }^{1}$ Azabu University \\ ${ }^{2}$ Kagawa Livestock Hospital \\ ${ }^{3}$ Nippon Veterinary and Life Science University
}

January 24, 2021

\begin{abstract}
Atypical porcine pestivirus (APPV), which has been confirmed to be associated with congenital tremor (CT) in pigs, is a newly discovered porcine virus that has been found in the Americas, Europe, and Asia; however, no report of APPV in Japan has been published. We identified an APPV in the central nervous system of Japanese piglets with CT, and firstly determined and analyzed the complete genome sequence. Phylogenetic analysis using the complete genome nucleotide sequence of the Japanese APPV, named Anna/2020, and those of APPVs from the NCBI database showed that APPVs were divided into three genotypes (genotypes 1 to 3), and that Anna/2020 clustered with the genotype 3 APPV strains, but distantly branched from these strains. Pairwise complete coding region nucleotide sequence comparisons revealed that there was $94.0 \%$ to $99.7 \%$ sequence identity among the genotype 3 strains, while Anna/2020 showed $87.0 \%$ to $89.3 \%$ identity to those genotype 3 strains, suggesting that Anna/2020 represents a novel APPV lineage within genotype 3. Retrospective examinations using RT-PCR revealed one genotype 1 and two novel genotype 3 APPVs from pigs without CT, and that novel genotype 3 APPVs have been prevalent in Japan since at least 2007.
\end{abstract}

Detection and genetic analysis of a novel atypical porcine pestivirus from piglets with congenital tremor in Japan

Short running title: Atypical porcine pestiviruses from pigs in Japan

Kasahara-Kamiie Miwako $^{1 *}$, Mitsuo Kagawa ${ }^{2 *}$, Mai Shiokawa ${ }^{3}$, Fujiko Sunaga ${ }^{1}$, Yuka Fukase ${ }^{1}$, Naoyuki Aihara $^{1}$, Takanori Shiga ${ }^{1}$, Junichi Kamiie ${ }^{1}$, Hiroshi Aoki ${ }^{3}$ and Makoto Nagai ${ }^{1}$

${ }^{*}$ Co-first authors.

${ }^{1}$ School of Veterinary Medicine, Azabu University, Sagamihara, Kanagawa 252-5201, Japan

${ }^{2}$ Kagawa Livestock Hospital, Annaka, Gunma 379-0133, Japan

${ }^{3}$ Faculty of Veterinary Science, Nippon Veterinary and Life Science University, Musashino, Tokyo 180-8602, Japan

Correspondence: Makoto Nagai, School of Veterinary Medicine, Azabu University, Sagamihara, Kanagawa 252-5201, Japan. Emil:m-nagai@azabu-u.ac.jp

\section{Summary}


Atypical porcine pestivirus (APPV), which has been confirmed to be associated with congenital tremor (CT) in pigs, is a newly discovered porcine virus that has been found in the Americas, Europe, and Asia; however, no report of APPV in Japan has been published. We identified an APPV in the central nervous system of Japanese piglets with CT, and firstly determined and analyzed the complete genome sequence. Phylogenetic analysis using the complete genome nucleotide sequence of the Japanese APPV, named Anna/2020, and those of APPVs from the NCBI database showed that APPVs were divided into three genotypes (genotypes 1 to 3), and that Anna/2020 clustered with the genotype 3 APPV strains, but distantly branched from these strains. Pairwise complete coding region nucleotide sequence comparisons revealed that there was $94.0 \%$ to $99.7 \%$ sequence identity among the genotype 3 strains, while Anna/2020 showed $87.0 \%$ to $89.3 \%$ identity to those genotype 3 strains, suggesting that Anna/2020 represents a novel APPV lineage within genotype 3. Retrospective examinations using RT-PCR revealed one genotype 1 and two novel genotype 3 APPVs from pigs without CT, and that novel genotype 3 APPVs have been prevalent in Japan since at least 2007.

Key words: atypical porcine pestivirus, congenital tremor, Japan, novel genotype

\section{INTRODUCTION}

Congenital tremor (CT) in neonatal piglets, also known as "dancing piglet", was described for the first time in 1922 (Kinsley, 1922). At present, CT is divided into types A and B. Type A is associated with visible histological lesions in the central nervous system (CNS) while type B does not show any apparent lesions. Type $\mathrm{A}$ is further divided into types A-I to A-V based on the causative agent, e.g., classical swine fever virus infection (A-I), genetic defect in the Landrace (A-III) and Saddleback (A-IV) pig breeds, and metrifonate intoxication (A-V) (Done, 1976); however, the causal factor of type A-II CT remained unknown for a long time. Recently, several viruses have been proposed to cause type A-II CT, including atypical porcine pestivirus (APPV), and only APPV has been proven to induce CT through the injection of pregnant sows with sera containing APPV (Arruda et al., 2016; de Groof et al., 2016).

APPV belongs to the genus Pestivirus within the family Flaviviridae. Recently, pestiviruses were categorized into 11 different species based on phylogenetic analysis, and a new host-independent taxonomy system for Pestivirus species was proposed using the format Pestivirus $X$. APPV was categorized asPestivirus K (Smith et al., 2017). Pestivirus is a small enveloped virus that has a single-stranded positive-sense RNA genome and contains a large open reading frame (ORF), which encodes nearly 4,000 amino acids (aa) and is preceded by a 5' untranslated region (UTR) and followed by a 3' UTR. The polyprotein is processed into four structural proteins $\left(\mathrm{C}, \mathrm{E}^{\mathrm{rns}}, \mathrm{E} 1\right.$, and $\left.\mathrm{E} 2\right)$ and eight non-structural proteins ( $\mathrm{N}^{\text {pro }}, \mathrm{p} 7, \mathrm{NS} 2$, NS3, NS4A, NS4B, NS5A, and NS5B), and the gene order is 5'-N ${ }^{\text {pro }}-C_{-} E^{\text {rns }}-E 1-E 2-p 7-N S 2-3(N S 2-N S 3)-N S 4 A-N S 4 B-N S 5 A-N S 5 B-3$ ' (Tautz et al., 2015).

APPV was first discovered from the serum of a pig without clinical symptoms in the United States (Hause et al., 2015). Since then, APPVs have been found from pigs and wild boars with or without CT worldwide on four continents, and genomic sequence diversity among APPVs has been reported (Beer et al., 2017; Cagatay et al., 2018; Chen et al., 2019; Choe et al., 2020; Colom-Cadena et al., 2018; de Groof et al., 2016; Dénes et al., 2018; Dessureault et al., 2018; Gatto et al., 2018; Mósena et al., 2018; Muñoz-Gonzalez et al., 2017; Pan et al., 2019b; Possatti et al., 2018; Postel et al., 2016, 2017; Schwarz et al., 2017; Shen et al., 2018; Sozzi et al., 2019; Stenberg et al., 2020; Xie et al., 2019; Yan et al., 2019; Yuan et al., 2017; Zhang et al., 2017; Zhang et al., 2019; Zhou et al., 2019). However, genome sequence data of APPV have not been reported from Japan.

In the present study, we identified the APPV genome sequence in piglets showing CT on a Japanese pig farm, and we analyzed and compared the complete genome sequences obtained from Japanese pigs with sequences from the DDBJ/EMBL/GenBank databases. Furthermore, a retrospective study to detect APPV was conducted using a total of 399 porcine samples collected from 2005 to 2020 in Japan.

\section{MATERIALS AND METHODS}

2.1 Routine diagnosis 
Tissues of the CNS (cerebellum), mesenteric lymph node, and tonsil from two 3-day-old piglets that showed severe signs of CT and moribund were collected and examined by reverse transcription (RT)-polymerase chain reaction (PCR) for pathogens of neurological disorders, such as porcine teschovirus (Krumbholz et al., 2003), porcine sapelovirus (Zell et al., 2000), porcine astrovirus (Chu et al., 2008), and APPV (Postel et al., 2016), and by real-time RT-PCR (qRT-PCR) for porcine circovirus 2 (Sunaga et al., 2019), pseudorabies virus (Ma et al., 2008), porcine hemagglutinating encephalomyelitis virus (Sunaga et al., 2019), Haemophilus parasuis (Turni et al., 2010), andStreptococcus suis (Bonifait et al., 2014). Tissues were minced by scissors, diluted 1:10 in phosphate-buffered saline, and homogenized for $20 \mathrm{sec}$ at 3,200 rpm in the presence of three stainless steel beads $(\varphi 4 \mathrm{~mm})$ by using a bead crusher $\mu \mathrm{T}-12$ (TAITEC, Inc.). To obtain the supernatant, organ emulsions were centrifuged at $12,000 \mathrm{~g}$ for $5 \mathrm{~min}$. Viral RNA and DNA and bacterial DNA were extracted from the supernatant using an IndiSpin Pathogen Kit (Indical Bioscience). For conventional RTPCR, cDNA synthesis was carried out using PrimeScript RT Master Mix (Takara Bio). PCR was performed using GoTaq® G2 Hot Start Taq Polymerase (Promega). qRT-PCR and PCR were performed using a One Step PrimeScript RT-PCR Kit (Takara Bio) for RNA viruses and Premix Ex Taq (Takara Bio) for DNA viruses and bacteria, and performed as described previously (Sunaga et al., 2019).

\subsection{Pathological examination}

The cerebrum, medulla oblongata, lymph nodes, and spleen of the two CT-affected piglets that were examined by RT-PCR were fixed with $10 \%$ buffered formalin, and embedded in paraffin by a conventional method. Sections were prepared from the blocks and stained with hematoxylin and eosin.

2.3 RT-PCR, 5' rapid amplification of cDNA end (5'RACE), and sequencing for whole genome sequence determination

Total RNA was extracted from CNS tissue using a QIAamp viral RNA mini kit (Qiagen). RT-PCR was carried out as described above using the primers listed in Supplementary Figure 1. The amplification of the 5' end of the virus genome was performed by the RACE method using a commercial kit (Invitrogen) and three reverse primers, APPV5'R1, APPV5'R2, and APPV5'R3 (Supplementary Figure 1). The A-Plus Poly(A) Polymerase Tailing Kit (CELLSCRIPT) and a reverse primer, TX30SXN (Oka et al., 2017), were employed for the amplification of the 3' end of the APPV genome.

\subsection{Retrospective investigation}

A retrospective investigation for the detection of APPV RNA was performed using a total of 399 samples; they consisted of the supernatants of homogenized organ tissues or sera that were collected from 1-day-old to 3-year-old pigs and had been submitted to the Pig Clinical Center in Azabu University for the diagnosis of diseases from 2005 to 2020. Viral RNA was extracted using an IndiSpin Pathogen Kit or QIAamp viral RNA mini kit. cDNA was synthesized using PrimeScript RT Master Mix. SYBR Green qRT-PCR was carried out using FastStart Essential DNA Green Master (Roche Diagnostics) with the primer pair reported by Postel et al. (2016). qRT-PCR-positive samples were subjected to amplification of the whole genome through conventional RT-PCR using the primers listed in Supplementary Figure 1, and by Sanger sequencing.

\subsection{Genome analysis}

Phylogenetic analysis using the complete nucleotide (nt) sequences of APPV was performed by the maximum likelihood method with the best fit model (GTR+G+I) in MEGA7 (Kumar et al., 2016). For the maximum likelihood tree analyses of the partial genome of 5'UTR-N pro (using $329 \mathrm{nt}), \mathrm{E}^{\mathrm{rns}}-\mathrm{E} 1$ (197 nt), NS3 (159 nt), NS5b (330 nt), and NS5b (258 nt), K2+G, T92+G+I, K2+G, TN93+I, and K2+G+I were used for the best fit models. Pairwise nt sequence identities were calculated using CLC Genomics Workbench 7.5.5 (CLC Bio). Similarity plot analysis was performed using SimPlot software V.3.5.1 (Lole et al., 1999).

\section{RESULTS}

3.1 Clinical history and routine detection of potential swine pathogens 
In March 2020, 15 out of approximately 150 litters in a commercial pig farm, which kept approximately 800 breeding crossbred sows, on the main island of Japan were affected by CT. Approximately $80 \%$ to $90 \%$ of the piglets in each litter showed varying degrees of repetitive constant tremors of the body. Severely affected piglets with CT displayed systemic tremors, had difficulty standing and sucking milk, and died, whereas piglets with mild clinical symptoms recovered and grew normally. The pre-weaning mortality rates in February, March, and April of 2020 were $6.0 \%, 5.1 \%$, and 5.4\%, respectively. On this farm, pigs with CT have been sporadically observed from about 15 years prior.

All samples from the two piglets with severe CT were negative for porcine teschovirus, porcine sapelovirus, porcine astrovirus, porcine circovirus 2, pseudorabies virus, hemagglutinating encephalomyelitis virus, and H. parasuis, but were positive for APPV and S. suis (data not shown).

\subsection{Pathological examination}

Histopathological investigations revealed many vacuoles in the medulla oblongata (Figure 1a) and the white matter around the ventriculus lateralis of the cerebrum (Figure 1b) in one of the two piglets examined. There was no inflammatory reaction observed as perivascular infiltration. No lesion was observed in the cerebrum or medulla oblongata of the other piglet. In both piglets, a decrease in lymphocytes as well as follicular atrophy in the lymph nodes and spleen were seen.

\subsection{Analysis of the whole genome sequence of the Japanese APPV}

Due to the lack of available Japanese APPV genome data in the NCBI database, we determined the complete genome sequence of this APPV, which was named Anna/2020, through RT-PCR and Sanger sequencing. We successfully determined the complete genome consisting of 11,567 nt sequences (Figure 2a). Anna/2020 possessed an ORF, which encoded a protein of 3,635 aa, flanked by a 5'UTR of $378 \mathrm{nt}$ and a 3'UTR of $281 \mathrm{nt}$. Phylogenetic tree analysis using the complete genome nt sequences of Anna/2020 and APPVs from the NCBI database showed that APPVs were clearly divided into three genotypes (Figure 2c). Genotype 1 consisted of APPVs from the Americas, Europe, and Asia, while genotypes 2 and 3 contained only Chinese APPVs. Anna/2020 falls into genotype 3 with the Chinese APPVs; however, it branched independently and distantly from the Chinese APPVs (Figure 2c). Pairwise sequence identity analysis of the complete coding regions revealed that Anna/2020 exhibited $80.2 \%$ to $81.8 \%$ and $80.9 \%$ to $81.2 \%$ sequence identity to the genotype 1 and genotype 2 APPVs. While there is $94.0 \%$ to $99.7 \%$ sequence identity among the Chinese APPVs in genotype 3, Anna/2020 showed $87.0 \%$ to $89.3 \%$ identity to these Chinese APPVs in genotype 3 (Table 1). Recombination events are an important mechanism by which viruses acquire genetic diversity, and they have been identified in APPVs (Guo et al., 2020; Xiao et al., 2016). Thus, to find crossover sites between Anna/2020 and other APPVs, similarity plot analysis was performed using SimPlot software. Anna/2020 exhibited higher sequence homology to genotype 3 APPVs than to genotype 1 and 2 APPVs throughout the whole genome, and no recombination event was observed (Figure $2 \mathrm{~b}$ ).

\subsection{Retrospective identification of APPV RNAs}

A total of 399 samples obtained from pigs during 2005 to 2020 were subjected to SYBR Green qRT-PCR to detect APPV RNA. The results showed that three samples were positive; these samples were collected from 20-day-old, 68-day-old, and 12-week-old pigs without CT that were kept in Kyusyu in 2007, the main island in 2018, and Kyusyu in 2018, respectively (Figure 3). Although we tried to determine the whole genome sequences of these samples, we could obtain only partial genome sequences, probably due to the low quality and insufficient amount of samples. The obtained partial sequences were aligned to the complete genome sequence of Anna/2020 (Figure 4a). Similarity and phylogenetic analysis using the APPV sequences from the NCBI database revealed that two APPVs from the samples collected in Kyusyu in 2007 and 2018, named Miya/2007 and Kago/2018, respectively, were assigned to genotype 3, and they formed a lineage with Anna/2020 that was distant from the Chinese APPVs in the phylogenetic trees; in contrast, all analyzed regions of the APPV detected in the sample collected on the main island in 2018, named Mae/2018, belonged to genotype 1 (Figure $4 \mathrm{~b}$ and $\mathrm{c}$ ). 


\section{DISCUSSION}

The mortality rates of CT clinical disease caused by APPV are generally low, and the economic losses remain unknown; however, severely affected piglets with CT may die due to colostrum intake difficulties and starvation, resulting in a higher pre-weaning mortality rate (Pan et al., 2019a; Stenberg et al., 2020). Clinical signs of CT have been observed since 15 years ago on the farm where Anna/2020 was detected, so the presence of APPV in the piglets might have been causing economic losses for the farm. A routine diagnosis revealed that S. suiswas simultaneously identified with APPV in the CNS of CT-affected piglets. The co-infection of APPV with other viruses, such as porcine circovirus 3 or teschovirus, has been reported (Possatti et al., 2018; Yan et al., 2019). APPV RNA was frequently found in lymphoid organs, suggesting that APPV may play a role in suppressing the host immune system (Pan et al., 2019a). Immune suppression might facilitate the co-infection of APPV with S. suis in CT-affected piglets. The histopathological findings of atrophy and fewer lymphocytes in the spleen and lymph nodes of affected piglets suggested that they were under immunosuppressive conditions.

According to phylogenetic dendrograms and pairwise sequence comparisons, APPVs are subdivided into three genotypes (Choe et al., 2020; Folgueiras-Gonzalez et al., 2020; Guo et al., 2020; Xie et al., 2019; Yan et al., 2019). Our analyses showed that Anna/2020 belonged to genotype 3; however, Anna/2020 formed an independent branch, and was distantly related to the Chinese genotype 3 strains. Furthermore, Anna/2020 showed $87.0 \%$ to $89.3 \%$ identity in the complete coding sequences to the other genotype 3 APPVs, while there is $94.0 \%$ to $99.7 \%$ identity among the Chinese APPV genotype 3 strains. Thus, it is suggested that Anna/2020 constitutes a novel APPV lineage within genotype 3. APPV genotype 3 strains have been reported only from China (Guo et al., 2020; Xie et al., 2019; Yan et al., 2019); thus, Anna/2020 represents the first strain belonging to genotype 3 from a country other than China. Furthermore, no recombination event was found between Anna/2020 and other APPVs from the NCBI database. These findings suggest that Anna/2020 might have independently evolved only in the Japanese pig population. Interestingly, two Japanese APPVs identified in samples from 2007 and 2018 in the retrospective study branched with Anna/2020 and formed a cluster, indicating that the novel genotype 3 lineage of APPVs has been present in Japan since at least 2007. Genotype 3 APPVs were identified in pigs on the main island of Japan and on Kyusyu island, suggesting that the APPVs of the novel genotype 3 lineage may already be prevalent throughout Japan. Retrospective examination also showed the presence of a genotype 1 APPV in Japan. The genotype 1 APPV was identified on a farm in close proximity to the farm where genotype 3 Anna/2020 was detected (Figure 3). Further studies are warranted to clarify the prevalent APPV genotype(s) in Japan.

Three $(0.75 \%)$ APPV RNAs were found using qRT-PCR in 399 previously obtained samples from pigs without a history of CT. In recent APPV prevalence studies of apparently healthy pigs by qRT-PCR, the APPV detection rates were $2.3 \%$ to $22 \%$ in Europe, the United States, and Asia (Beer et al., 2017; Hause et al., 2015; Postel et al., 2016, 2017). Our detection rate was lower, and may be attributable to the low quality of the preserved samples or biased sample collection. A recent report showed that $19 \%$ of wild boars possessed APPV RNA, suggesting that the boars may be a source of APPV transmission (Cagatay et al., 2018). Further investigations on the prevalence of APPV in pigs and wild boars are needed for estimating the precise prevalence of APPV, and for establishing proper control and prevention measures for APPV infection in Japan.

In conclusion, in the present study, we firstly determined the complete genome sequence of an APPV detected from CT-affected piglets in Japan. Genomic analyses revealed that this strain is a novel APPV that forms a new lineage within genotype 3 APPVs. The retrospective study demonstrated that one genotype 1 and two novel genotype 3 APPVs were identified from pigs without CT, and the novel genotype 3 strains were closely related to Anna/2020. The novel genotype 3 lineage of APPV might have been prevalent in Japan since at least 13 years ago, and evolved only in the Japanese pig population.

\section{ACKNOWLEDGEMENTS}

Part of this work was carried out under an agreement on collaboration between Azabu University and 
the Nippon Veterinary and Life Science University, the JSPS KAKENHI (grant number 18K05977), and Grants-in-Aid from the Japan Racing Association and Livestock Promotion Foundation.

\section{CONFLICT OF INTEREST}

The authors have no conflicts of interest to declare.

\section{ETHICAL APPROVAL}

The authors confirm that the ethical policies of the journal, as noted in the journal's author guidelines, have been adhered to, and approval was received from the appropriate ethics review committee. No experimental animals were used for the reported work.

\section{DATA AVAILABILITY STATEMENT}

The data that support the findings of this study are available from the corresponding author upon reasonable request.

\section{REFERENCES}

Arruda, B. L., Arruda, P. H., Magstadt, D. R., Schwartz, K. J., Dohlman, T., Schleining, J. A., Patterson, A. R., Visek, C. A., \& Victoria, J. G., (2016). Identification of a Divergent Lineage Porcine Pestivirus in Nursing Piglets with Congenital Tremors and Reproduction of Disease following Experimental Inoculation. PLoS One, 24;11(2):e0150104. https://doi.org/10.1371/journal.pone.0150104.

Beer, M., Wernike, K., Drager, C., Hoper, D., Pohlmann, A., Bergermann, C., Schroder, C., Klinkhammer, S., Blome, S., \& Hoffmann, B., (2017).Transboundary and emerging diseases , 64, e22e26.https://doi.org/10.1111/tbed.12532.

Bonifait, L., Veillette, M., Letourneau, V., Grenier, D. and Duchaine, C., (2014). Detection of streptococcus suis in bioaerosols of swine confinement buildings. Applied and environmental microbiology , 80, 3296-3304. https://doi.org/10.1128/AEM.04167-13.

Cagatay, G. N., Antos, A., Meyer, D., Maistrelli, C., Keuling, O., Becher, P., \& Postel, A., (2018). Frequent infection of wild boar with atypical porcine pestivirus (APPV). Transboundary and emerging diseases , 65, 1087-1093. https://doi.org/10.1111/tbed.12854.

Chen, F., Knutson, T. P., Braun, E., Jiang, Y., Rossow, S., \& Marthaler, D. G., (2019). Semiquantitative duplex RT-PCR reveals the low occurrence of Porcine Pegivirus and Atypical Porcine Pestivirus in diagnostic samples from the United States.Transboundary and emerging diseases , 66, 1420-1425. https://doi.org/10.1111/tbed.13154.

Choe, S., Park, G. N., Cha, R. M., Hyun, B. H., Park, B. K., \& An, D. J. (2020). Prevalence and genetic diversity of atypical porcine pestivirus (APPV) detected in South Korean wild boars. Viruses , 24;12(6):680.https://doi.org/10.3390/v12060680.

Colom-Cadena, A., Ganges, L., Munoz-Gonzalez, S., Castillo-Contreras, R., Bohorquez, J. A., Rosell, R., Segales, J., Marco, I., \& Cabezon, O., (2018). Atypical porcine pestivirus in wild boar (Sus scrofa), Spain. The Veterinary record, 183, 569. https://doi: 10.1136/vr.104824.

de Groof, A., Deijs, M., Guelen, L., van Grinsven, L., van Os-Galdos, L., Vogels, W., Derks, C., Cruijsen, T., Geurts, V., Vrijenhoek, M., Suijskens, J., van Doorn, P., van Leengoed, L., Schrier, C., \& van der Hoek, L., (2016). Atypical Porcine Pestivirus: A Possible Cause of Congenital Tremor Type A-II in Newborn Piglets. Viruses , 4;8(10):271. https://doi.org/10.3390/v8100271.

Denes, L., Biksi, I., Albert, M., Szeredi, L., Knapp, D. G., Szilasi, A., Balint, A., \& Balka, G., (2018). Detection and phylogenetic characterization of atypical porcine pestivirus strains in Hungary. Transboundary and emerging diseases , 65, 2039-2042.https://doi.org/10.1111/tbed.12981. 
Dessureault, F. G., Choiniere, M., Provost, C., \& Gagnon, C. A., (2018). First report of atypical porcine pestivirus in piglets with congenital tremor in Canada. The Canadian veterinary journal , 59, 429-432.

Done, J. T. (1976). The congenital tremor syndrome in piglets. Veterinary Annual , 16, 98-102.

Gatto, I. R. H., Harmon, K., Bradner, L., Silva, P., Linhares, D. C. L., Arruda, P.H., de Oliveira, L. G., \& Arruda, B. L., (2018). Detection of atypical porcine pestivirus in Brazil in the central nervous system of suckling piglets with congenital tremor. Transboundary and emerging diseases , 65, 375380.https://doi.org/10.1111/tbed.12824.

Guo, Z., Wang, L., Qiao, S., Deng, R., \& Zhang, G., (2020). Genetic characterization and recombination analysis of atypical porcine pestivirus. Infection, Genetics and Evolution , 81, 104259. https://doi.org/10.1016/j.meegid.2020.104259.

Hause, B. M., Collin, E. A., Peddireddi, L., Yuan, F., Chen, Z., Hesse, R. A., Gauger, P. C., Clement, T., Fang, Y., \& Anderson, G. (2015). Discovery of a novel putative atypical porcine pestivirus in pigs in the USA. Journal of general virology , 96, 2994-2998. https://doi.org/10.1099/jgv.0.000251.

Kinsley, A.T. (1922). Dancing pigs? Veterinary Medicine, 17, 123.

Kumar. S., Stecher, G., \& Tamura, K., (2016). MEGA7: Molecular Evolutionary Genetics Analysis version 7.0 for bigger datasets.Molecular biology and evolution , 33. 1870-1874. https://doi.org/10.1093/molbev/msw054.

Krumbholz, A., Wurm, R., Scheck, O., Birch-Hirschfeld, E., Egerer, R., Henke, A., Wutzler, P., \& Zell, R., (2003). Detection of porcine teschoviruses and enteroviruses by LightCycler real-time PCR. Journal of virological methods, 113, 51-63.https://doi.org/10.1016/s0166-0934(03)00227-1.

Lole, K. S., Bollinger, R. C., Paranjape, R. S., Gadkari, D., Kulkarni, S. S., Novak, N. G., Ingersoll, R., Sheppard, H. W., \& Ray, S. C., (1999). Full-length human immunodeficiency virus type 1 genomes from subtype C-infected seroconverters in India, with evidence of intersubtype recombination. Journal of Virology , 73, 152-160. https://doi.org/10.1128/JVI.73.1.152-160.1999.

Ma, W., Lager, K. M., Richt, J. A., Stoffregen, W. C., Zhou, F., Yoon, K. J., (2008). Development of real-time polymerase chain reaction assays for rapid detection and differentiation of wild-type pseudorabies and gene-deleted vaccine viruses. Journal of veterinary diagnostic investigation , 20, 440-447. https://doi.org/10.1177/104063870802000405.

Mosena, A. C. S., Weber, M. N., da Cruz, R. A. S., Cibulski, S. P., da Silva, M. S., Puhl, D. E., Hammerschmitt, M. E., Takeuti, K. L., Driemeier, D., de Barcellos, D. E. S. N., \& Canal, C. W., (2018). Presence of atypical porcine pestivirus (APPV) in Brazilian pigs. Transboundary and emerging diseases , 65, 22-26.https://doi.org/10.1111/tbed.12753.

Munoz-Gonzalez, S., Canturri, A., Perez-Simo, M., Bohorquez, J. A., Rosell, R., Cabezon, O., Segales, J., Domingo, M., \& Ganges, L., (2017). First report of the novel atypical porcine pestivirus in Spain and a retrospective study. Transboundary and emerging diseases ,64, 1645-1649.https://doi.org/10.1111/tbed.12699.

Oka, T., Doan, Y. H., Shimoike, T., Haga, K., Takizawa, T., (201). First complete genome sequences of genogroup $\mathrm{V}$, genotype 3 porcine sapoviruses: common 5'-terminal genomic feature of sapoviruses. Virus Genes , 53, 848-855.https://doi.org/10.1007/s11262-017-1481-8.

Pan, S., Mou, C., \& Chen, Z., (2019a). An emerging novel virus: Atypical porcine pestivirus (APPV). Rev Med Virol. 2019 Jan;29(1):e2018. https://doi.org/10.1002/rmv.2018.

Pan, S., Yan, Y., Shi, K., Wang, M., Mou, C., \& Chen, Z., (2019b). Molecular characterization of two novel atypical porcine pestivirus (APPV) strains from piglets with congenital tremor in China. Transboundary and emerging diseases , 66, 35-42.https://doi.org/10.1111/tbed.13029. 
Possatti, F., Headley, S. A., Leme, R. A., Dall Agnol, A. M., Zotti, E., de Oliveira, T. E.S., Alfieri, A. F., \& Alfieri, A. A., (2018). Viruses associated with congenital tremor and high lethality in piglets. Transboundary and emerging diseases , 65, 331-337.https://doi.org/10.1111/tbed.12807.

Postel, A., Hansmann, F., Baechlein, C., Fischer, N., Alawi, M., Grundhoff, A., Derking, S., Tenhundfeld, J., Pfankuche, V. M., Herder, V., Baumgartner, W., Wendt, M., \& Becher, P., (2016). Presence of atypical porcine pestivirus (APPV) genomes in newborn piglets correlates with congenital tremor. Scientific reports , 13;6:27735. https://doi.org/10.1038/srep27735.

Postel, A., Meyer, D., Cagatay, G. N., Feliziani, F., De Mia, G. M., Fischer, N., Grundhoff, A., Milićević, V, Deng, M. C., Chang, C. Y., Qiu, H. J., Sun, Y, Wendt, M., \& Becher, P., (2017). High Abundance and Genetic Variability of Atypical Porcine Pestivirus in Pigs from Europe and Asia. Emerging infectious diseases , 23, 2104-2107. https://doi.org/10.3201/eid2312.170951.

Schwarz, L., Riedel, C., Högler, S., Sinn, L. J., Voglmayr, T., Wöchtl, B., Dinhopl, N., Rebel-Bauder, B., Weissenböck, H., Ladinig, A., Rümenapf, T., \& Lamp, B., (2017). Congenital infection with atypical porcine pestivirus (APPV) is associated with disease and viral persistence. Veterinary research , 6;48(1):1. https://doi.org/10.1186/s13567-016-0406-1.

Shen, H., Liu, X., Zhang, P., Wang, L., Liu, Y., Zhang, L., Liang, P., \& Song, C., (2018). Identification and characterization of atypical porcine pestivirus genomes in newborn piglets with congenital tremor in China. Journal of veterinary science, 31;19(3):468-471. https://doi.org/10.4142/jvs.2018.19.3.468.

Smith, D. B., Meyers, G., Bukh, J., Gould, E.A., Monath, T., Scott Muerhoff, A., Pletnev, A., Rico-Hesse, R., Stapleton, J. T, Simmonds, P., \& Becher, P. (2017). Proposed revision to the taxonomy of the genus Pestivirus, family Flaviviridae. Journal of general virology , 98, 2106-2112. https://doi.org/10.1099/jgv.0.000873.

Sozzi, E., Salogni, C., Lelli, D., Barbieri, I., Moreno, A., Alborali, G. L., \& Lavazza, A. (2019). Molecular Survey and Phylogenetic Analysis of Atypical Porcine Pestivirus (APPV) Identified in Swine and Wild Boar from Northern Italy. Viruses , 10;11(12):1142. https://doi.org/10.3390/v11121142.

Stenberg, H., Jacobson, M., \& Malmberg, M. (2020). Detection of atypical porcine pestivirus in Swedish piglets with congenital tremor type A-II. BMC veterinary research . 29;16(1):260.https://doi.org/10.1186/s12917-020-02445-w.

Sunaga, F., Tsuchiaka, S., Kishimoto, M., Aoki, H., Kakinoki, M., Kure, K., Okumura, H., Okumura, M., Okumura, A., Nagai, M., Omatsu, T., \& Mizutani, T., (2019). Development of a one-run real-time PCR detection system for pathogens associated with porcine respiratory diseases. The Journal of veterinary medical science , 82, 217-223. https://doi.org/10.1292/jvms.19-0063.

Tautz, N., Tews, B. A., \& Meyers, G. (2015). The molecular biology of pestiviruses. Advances in virus research ,93, 47-160.https://doi.org/10.1016/bs.aivir.2015.03.002.

Turni, C., Pyke, M. and Blackall, P. J., (2010). Validation of a real-time PCR for Haemophilus parasuis. Journal of applied microbiology , 108, 1323-1331.https://doi.org/10.1111/j.1365-2672.2009.04526.x.

Xiao, Y., Rouzine, I. M., Bianco, S., Acevedo, A., Goldstein, E. F., Farkov, M., Brodsky, L., \& Andino, R., (2016). RNA recombination enhances adaptability and is required for virus spread and virulence. Cell host and microbe , 13;19(4):493-503. https://doi.org/10.1016/j.chom.2016.03.009.

Xie, Y., Wang, X., Su, D., Feng, J., Wei, L., Cai, W., Li, J., Lin, S., Yan, H., He, \& D., (2019). Detection and genetic characterization of atypical porcine pestivirus in piglets with congenital tremors in southern China. Frontiers in microbiology . 20;10:1406. https://doi.org/10.3389/fmicb.2019.01406.

Yan, X. L., Li, Y. Y., He, L. L., Wu, J. L., Tang, X. Y., Chen, G. H., Mai, K. J., Wu, R. T., Li, Q. N., Chen, Y. H., Sun, Y., \& Ma, J. Y. (2019). 12 novel atypical porcine pestivirus genomes from neonatal piglets with congenital tremors: A newly emerging branch and high prevalence in China. Virology , 533, 50-58. https://doi.org/ 10.1016/j.virol.2019.04.010. 
Yuan, J., Han, Z., Li, J., Huang, Y., Yang, J., Ding, H., Zhang, J., Zhu, M., Zhang, Y., Liao, J., Zhao, M., \& Chen, J., (2017). Atypical Porcine Pestivirus as a Novel Type of Pestivirus in Pigs in China.Frontiers in microbiology . 11;8:862. https://doi.org/10.3389/fmicb.2017.00862.

Zhang, X., Dai, R., Li, Q., Zhou, Q., Luo, Y., Lin, L., Bi, Y., \& Chen, F. (2019). Detection of three novel atypical porcine pestivirus strains in newborn piglets with congenital tremor in southern China.Infection, Genetics and Evolution , 68, 54-57. https://doi.org/10.1016/j.meegid.2018.12.008.

Zhou, K., Yue, H., Tang, C., Ruan, W., Zhou, Q., \& Zhang, B., (2019). Prevalence and genome characteristics of atypical porcine pestivirus in southwest China. Journal of general virology , 100, 8488.https://doi.org/10.1099/jgv.0.001188.

Zhang, K., Wu, K., Liu, J., Ge, S., Xiao, Y., Shang, Y., \& Ning, Z., (2017). Identification of atypical porcine pestivirus infection in swine herds in China. Transboundary and emerging diseases , 64, 1020-1023. https://doi.org/10.1111/tbed.12659.

\section{Figure legends}

FIGURE 1 (a) Histological findings of the medulla oblongata of a piglet with CT. Hematoxylin and eosin (HE) stain. The scale bar represents $200 \mu \mathrm{m}$. (b) Histological findings of the white matter around the ventriculus lateralis of the cerebrum of a piglet with CT. HE stain. The scale bar represents $100 \mu \mathrm{m}$.

FIGURE 2 (a) Schematic diagram showing the genome organization of Anna/2020. (b) Similarity plots of the complete genomes of genotype 1 APPVs (green lines), genotype 2 APPVs (blue lines), and genotype 3 APPVs (pink lines) with Anna/2020 as the query sequence using a sliding window of $200 \mathrm{nt}$ and a moving step size of 20 nt. (c) The phylogenetic tree was constructed using the maximum likelihood method (GTR+G+I model) in MEGA7 with 1000 bootstrap replicates. The scale bar indicates the corrected genetic distance. Anna/2020 is indicated in red text. The percent bootstrap support is indicated by the value at each node; values $<70 \%$ are omitted.

FIGURE 3 Distribution and occurrence of atypical porcine pestivirus infection or detection of atypical porcine pestivirus in Japan.

FIGURE 4 (a) Schematic diagram showing the genome organization of Anna/2020 and regions of the obtained genome sequences of Miya/2007, Kago/2018, and Mae/2018. The genome sequence positions of each strain corresponding to those of Anna/2020. (b) Similarity plots of the complete genomes of genotype 1 APPVs (green lines), genotype 2 APPVs (blue lines), genotype 3 APPVs (pink lines), and the partial genomes of Miya/2007 (brown lines), Kago/2018 (purple lines), and Mae/2018 (dark green lines) with Anna/2020 as the query sequence using a sliding window of $200 \mathrm{nt}$ and a moving step size of $20 \mathrm{nt}$. (c) The phylogenetic trees were constructed using the partial genome sequences of five regions by the maximum likelihood method in MEGA7 with 1000 bootstrap replicates. The scale bar indicates the corrected genetic distance. Anna/2020, Miya/2007, Kago/2018, and Mae/2018 are indicated in red, brown, purple, and dark green texts, respectively. The percent bootstrap support is indicated by the value at each node; values $<70 \%$ are omitted.

\section{Hosted file}

Figure. 1. Kasahara-Kamiie et al.20210111.pptx available at https://authorea.com/users/ 391388/articles/505516-detection-and-genetic-analysis-of-a-novel-atypical-porcinepestivirus-from-piglets-with-congenital-tremor-in-japan

\section{Hosted file}

Figure. 2. Kasahara-Kamiie et al.20201226.pptx available at https://authorea.com/users/ 391388/articles/505516-detection-and-genetic-analysis-of-a-novel-atypical-porcinepestivirus-from-piglets-with-congenital-tremor-in-japan

\section{Hosted file}


Figure. 3. Kasahara-Kamiie et al.20201223.pptx available at https://authorea.com/users/ 391388/articles/505516-detection-and-genetic-analysis-of-a-novel-atypical-porcinepestivirus-from-piglets-with-congenital-tremor-in-japan

\section{Hosted file}

Figure. 4. Kasahara-Kamiie et al.20201227.pptx available at https://authorea.com/users/ 391388/articles/505516-detection-and-genetic-analysis-of-a-novel-atypical-porcinepestivirus-from-piglets-with-congenital-tremor-in-japan

\section{Hosted file}

Kasahara-Kamiie et al. Table 1 20210119.xlsx available at https://authorea.com/users/391388/ articles/505516-detection-and-genetic-analysis-of-a-novel-atypical-porcine-pestivirusfrom-piglets-with-congenital-tremor-in-japan 